\title{
Lumen degradation modeling of white-light LEDs in step stress accelerated degradation test
}

\author{
Jianlin Huang, Dušan S Golubović, Sau Koh, Daoguo Yang, Xiupeng Li, Xuejun Fan, G.Q. Zhang
}

\begin{abstract}
In this paper, lumen degradation is described by using a modified Brownian motion process for mid-power white-light LED packages, which were aged under step stress accelerated degradation test (SSADT). First, a SSADT model has been established based on the theory of equivalent accumulative damage. Then, a method was proposed to improve the accuracy of the parameter estimation by carefully modifying the estimator, which was proposed in the previous research. Experimental data show that parameters estimated by using SSADT model are very close to those estimated by using constant stress accelerated degradation test (CSADT) model, indicating the feasibility of the SSADT model. The experiment also indicates that SSADT can be used as an alternative to CSADT, as it enables comparable estimation accuracy, while using less testing time, a smaller sample size and less test capacity.
\end{abstract}

Index Terms - accelerated test; Brownian motion; degradation test; light-emitting diodes; step stress; Wiener process

\section{INTRODUCTION}

$\mathrm{M}$ ID-POWER white-light LEDs (MP-LEDs) have been widely considered as the new generation of luminaires due to their advantages of higher efficiency, longer lifetime, and more environment protections as compared to currently widely used lighting solutions (incandescence, fluorescence). Recently, due to fast evolution of the technologies, the lifetime of LED packages has been claimed to be as high as 50,000 hours. For such kind of products, failed units would be seldom observed during life tests, even though they are subjected to accelerated conditions. Therefore, it is unreasonable to collect

Manuscript received January 04, 2016.

Jianlin Huang is with Beijing Research Center, Delft University of Technology, Beijing, 100083, China (phone: +86 150-0023-8137; fax: +86-21-5445-1520; e-mail: J.Huang-37@tudelft.nl).

D. S. Golubovi'c is with the Lumileds Commercial (Shanghai) Co., Ltd, Shanghai 200233, China (e-mail: dusan.golubovic@lumileds.com).

Sau Koh was with Beijing Research Center, Delft University of Technology, Beijing, 100083, China. He is now with Huawei Technologies Co., Ltd, Shenzhen, China. (E-mail: saukoh@ @klssl.org).

Daoguo Yang is with Guilin University of Electronic Technology, Guangxi, 541004, China (E-mail: d.g.yang @ guet.edu.cn).

Xiupeng Li is with Philips Lighting, Shanghai, 200233, China (E-mail: Xiupeng.Li@ @hilips.com).

Xuejun Fan is with State Key Laboratory of Solid State Lighting, Beijing 100083, PR China. He is also with Lamar University, Beaumont, Texas, 77710, USA (E-mail: xuejun.fan@lamar.edu).

G.Q. Zhang is with Delft University of Technology, 2628 CT, Delft, the Netherlands. He is also working for Institute of Semiconductors, Chinese Academy of Sciences, Beijing, 100083, PR China (E-mail: G.Q.Zhang@tudelft.nl). failure data for LED reliability analysis by performing accelerated life test (ALT) in which samples are generally recorded as time to failure.

Though catastrophic failures are seldom observed, LED packages showed gradual degradation during ageing tests. The degradation includes lumen decay, color shift $[1,2]$, and so forth. For these kinds of devices, it is possible to obtain degradation measurements over time, and these measurements may contain useful information about product reliability. By using these degradation data, the lifetime of LED packages can be estimated, according to the IES standards [3,4]. The test method and related data analysis techniques in these standards, are typically called accelerated degradation test (ADT), which have been studied for many years [5-7]. Researchers have also developed several statistic models for this kind of accelerated tests. These models are categorized into either general path model [8-11], or stochastic process model [12-16]. A review of the degradation modeling can been found in [17].

In general, constant stress accelerated degradation test (CSADT) and step stress accelerated degradation test (SSADT) are two of the most popular ADTs in industry applications. Compared to CSADT, SSADT enables comparable lifetime prediction accuracy while using smaller sample size and less test resources [18-20]. Therefore, the SSADT has been widely applied in reliability tests for LEDs [21, 22], transistors [23], and missile tanks [24]. Based on Nelson's equivalent accumulative damage theory, Tseng [18] and Yao [25], respectively, presented a segmented nonlinear accelerated degradation model (SNADM), which tackles the problem that the degradation rate of the products varies with time during SSADT. However, these models are regression-based and, thus, they cannot capture the dynamics or random variations of the degradation process. In order to solve this problem, Kjell et al. [26], Liao et al. [27], and Tsai et al. [28] proposed a stochastic-based SSADT model using Brownian motion and Gamma process. The stationary and independent incremental property of the stochastic processes renders simplicity of the modeling of SSADT.

The drawback of these stochastic models is that, they are only available for linear degradation behaviors, thus limiting the applications in a more broad class of degradation processes. Over the past years, researchers were making efforts to develop more flexible models for directly describing the nonlinear degradation through a stochastic process [29-33]. More recent works can be referred to the publications of Si et al. [34, 35], Wang et al. [36], Wang et al. [37, 38], Wang et al. [39], Huang et al. [40], Chiang et al. [41], . In these papers, researchers have 
modeled nonlinear degradation by using time-scaling techniques [37-39, 41], nonlinear drift coefficient [29-34], or an adaptive parameter which can be updated dynamically $[35,36$, 40]. While these models have only been developed for the degradation process in CSADT, there are few studies for the SSADT modeling, which use stochastic models to describe nonlinear degradation processes [27, 42].

In this paper, we presented a methodology for the SSADT modeling based on a modified Brownian motion. Firstly, the theory and characteristics of modified Brownian motion are introduced. Secondly, the SSADT model has been established by using the modified Brownian motion theory. Thirdly, a likelihood function was presented for the parameter estimation. Finally, a set of experiments, including CSADT and SSADT, were conducted on one type of mid-power white-light LED packages. After the experiments, parameter estimation was performed on data obtained from both SSADT and CSADT in order to verify the feasibility of our SSADT model.

\section{MODELING OF STEP STRESS ACCELERATED DEGRADATION TEST}

\section{A. Theory of Brownian motion process}

The modified Brownian motion $\{X(t), t \geq 0\}$ is defined as $[37,43]$ :

$$
X(t)=X(0)+\int_{0}^{t} \mu(t ; \theta) d t+\sigma B(t)
$$

where $\mu(t)$ is the drift rate, $\sigma$ is the diffusion coefficient, and $B(t)$ is the standard Brownian motion. Generally, the basic properties of Brownian motion could be characterized as follows:

(P1) The increment $\Delta X(t)=X(t+\Delta t)-X(t)$ is independent of $X(t)$, which means that if $0 \leq s_{1}<t_{1}<s_{2}<t_{2}$, then $X\left(t_{1}\right)-X\left(s_{1}\right)$ and $X\left(t_{2}\right)-X\left(s_{2}\right)$ are independent random variables, and the similar condition holds for $n$ increments;

$$
\text { (P2) } \Delta X(t) \sim N\left(\int_{s}^{s+t} \mu(\tau ; \theta) d \tau, \sigma^{2} t\right)
$$

where $\quad N\left(\int_{s}^{s+t} \mu(\tau ; \theta) d \tau, \sigma^{2} t\right)$ denotes the normal distribution with expected value $\int_{s}^{s+t} \mu(\tau ; \theta) d \tau$, and variance $\sigma^{2} t$

(P3) According to (P2), if $s=0$, $X(t) \sim N\left(\int_{0}^{t} \mu(t ; \theta) d t, \sigma^{2} t\right)$.

Assume under a certain stress level $S_{k}$, the LED's lumen degradation $X_{k}(t)$, can be described by Brownian motion. According to Eq. (1), we have

$$
X_{k}(t)=\int_{0}^{t} \mu_{k}(t ; \theta) d t+\sigma_{k} B(t)
$$

Given a threshold $w$, the LED is deemed to fail once the observed lumen degradation $X_{k}(t)$ crosses the specified threshold. From the first hitting time (FHT) concept, the lifetime $T$ can be defined as

$T=\inf \left\{t: X_{k}(t) \geq w \mid X_{k}(0)<w\right\}$.

By transforming the problem from calculating the FHT distribution of the nonlinear diffusion process crossing a constant threshold into a standard Brownian motion process crossing a time-dependent boundary, the probability distribution (PDF) of $X_{k}(t)$ crossing a constant boundary $w$ has been derived by $\mathrm{Si}$ et al. [34]. Furthermore, with the assumption that the probability of such a degradation process crosses the threshold level before time $t$ is negligible, the explicit form of an approximation to the PDF of the FHT distribution was given as follows [34]:

$$
\begin{aligned}
p_{X_{k}(t)}(w, t) \cong & \frac{1}{\sqrt{2 \pi t}}\left(\frac{S_{B}(t)}{t}+\frac{1}{\sigma_{k}} \mu_{k}(t ; \theta)\right) \\
& \cdot \exp \left[-\frac{S_{B}^{2}(t)}{2 t}\right], \\
\text { where } S_{B}(t)= & \frac{1}{\sigma_{k}}\left(w-\int_{0}^{t} \mu_{k}(\tau ; \theta) d \tau\right) .
\end{aligned}
$$

\section{B. SSADT modeling by using Brownian motion process}

SSADT is a special type of ADT in which stress levels are increased during the test period in a specified discrete sequence. A typical stress pattern is shown in Fig. 1. In this paper, the SSADT has been performed based on following assumptions:

(A1) In all accelerated stress levels, the failure modes and failure mechanisms are the same;

(A2) Testing data measured at each readout point in each stress level follow normal distribution;

(A3) The remaining life of the samples only depend on the currently failed cumulative fraction and current stress, and

(A4) The total degradation $\Delta D_{T}(t)$ induced by any combination of stress levels is equivalent to the degradation induced by a single stress level $S_{k}$, so $\Delta D_{T}(t)=\int_{0}^{\tau_{k}} \mu_{k}(\tau ; \theta) d \tau$, where $\tau_{k}$ is the equivalent test time under stress $S_{k}$.

When designing an SSADT plan, it is important to ensure that the degradation mechanisms are consistent in all stress sequences. In fact, this is one of the radical rules for all accelerated tests, including CASDT and SSADT. In general, this is guaranteed empirically, based on the products' properties and their practical operating conditions. For instance, an LED package may be recommended to be operated with its junction temperature lower than $125{ }^{\circ} \mathrm{C}$. On the other hand, a few techniques were also proposed for the judgment of mechanism consistency in accelerated tests. They are - statistic distribution analysis [44], activation energy calculation [45, 46], and independent parameters in degradation models $[18,19]$. 
(a) Single stress factor

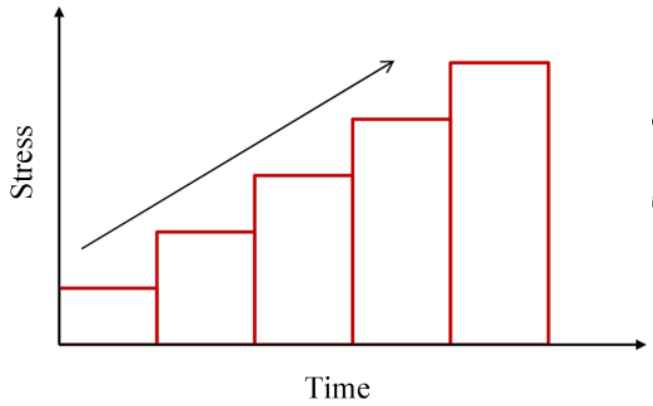

(b) Dual stress factors

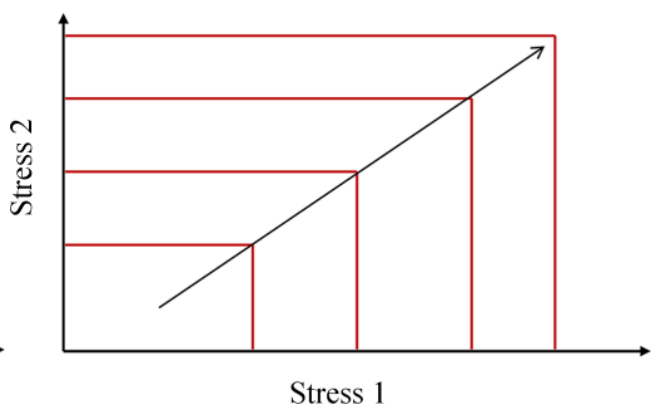

Fig. 1 Step stress pattern with different stress factors

For assumption (A2), it can be easily verified after all test data are obtained. For assumptions (A3) and (A4), it is actually based on Nelson's equivalent accumulative damage theory [47], which has widely been used in step stress accelerated tests.

Assume $N$ units of samples are tested under a k-step stress accelerated test, and each sample is measured $M_{k}$ times at the $k^{\text {th }}$ stress level. Under each stress level, the measurement point $M_{k}$ is allowed to be different. Without loss of generality, we assume that the stress series are $S_{1}, S_{2}, \cdots, S_{k}$, and $t_{k+1}$ is the time point at which the stress changes from the $k^{\text {th }}$ stress level to the $(k+1)^{t h}$ stress level. Thus, $t_{1}=0$, and $t_{k+1}$ is the end time of the test.

For a k-step stress accelerated test, define $\Delta D\left(t ; S_{k}\right)$ as the lumen degradation of LED package induced by stress $S_{k}$ after time $t$. Based on assumption (A4), we have

$$
\begin{aligned}
& \int_{t_{1}}^{t} \mu_{1}(t ; \theta) d t=\Delta D\left(t-t_{1} ; S_{1}\right), \quad t_{1}=0 . \\
& \int_{\tau_{k}}^{\tau_{k}+\left(t-t_{k}\right)} \mu_{k}(t ; \theta) d t=\Delta D\left(t-t_{k} ; S_{k}\right) .
\end{aligned}
$$

From Eq. (5) and (6), the total degradation $\Delta D_{T}(t)$ of an LED package after a k-step stress accelerated test can be obtained as:

$$
\begin{aligned}
\Delta D_{T}(t) & =\int_{t_{1}}^{t_{2}} \mu_{1}(t ; \theta) d t+\int_{\tau_{2}}^{\tau_{2}+t_{3}-t_{2}} \mu_{2}(t ; \theta) d t \cdots \\
& +\int_{\tau_{k}}^{\tau_{k}+t-t_{k}} \mu_{k}(t ; \theta) d t .
\end{aligned}
$$

More specifically, Eq. (7) is rewritten as :

$$
\Delta D_{T}(t)=\left\{\begin{array}{cc}
\int_{t_{1}}^{t} \mu_{1}(t ; \theta) d t, & 0=t_{1} \leq t<t_{2} \\
\int_{0}^{\tau_{2}} \mu_{2}(t ; \theta) d t+\int_{\tau_{2}}^{\tau_{2}+\left(t-t_{2}\right)} & \mu_{2}(t ; \theta) d t, \\
\ldots & t_{2} \leq t<t_{3}, \\
\int_{0}^{\tau_{k}} \mu_{k}(t ; \theta) d t+\int_{\tau_{k}}^{\tau_{k}+\left(t-t_{k}\right)} & \mu_{k}(t ; \theta) d t, \\
& t_{k} \leq t<t_{k+1}
\end{array}\right.
$$

where

$$
\begin{aligned}
\int_{0}^{\tau_{k}} \mu_{k}(t ; \theta) d t=\int_{t_{1}}^{t_{2}} \mu_{1}(t ; \theta) d t & +\int_{\tau_{2}}^{\tau_{2}+t_{3}-t_{2}} \mu_{2}(t ; \theta) d t \cdots \\
& +\int_{\tau_{k-1}}^{\tau_{k-1}+t_{k}-t_{k-1}} \mu_{k-1}(t ; \theta) d t .
\end{aligned}
$$

Then according to Eq. (2), the stochastic process of lumen degradation can be written as:

$X(t)=\left\{\begin{array}{cr}\int_{0}^{t} \mu_{1}(t ; \theta)+\sigma_{1} B(t), \quad 0=t_{1} \leq t<t_{2} \\ \int_{0}^{\tau_{2}+\left(t-t_{2}\right)} \mu_{2}(t ; \theta) d t+\sigma_{2} B\left(\tau_{2}+t-t_{2}\right), \\ \ldots & t_{2} \leq t<t_{3} \\ \int_{0}^{\tau_{k}+\left(t-t_{k}\right)} \mu_{k}(t ; \theta) d t+\sigma_{k} B\left(\tau_{k}+t-t_{k}\right), \\ & t_{k} \leq t<t_{k+1} .\end{array}\right.$

\section{Parameter estimation}

Assume that $X_{i j k}$ is the $j^{\text {th }}$ degradation readout of the $i^{\text {th }}$ unit of the samples under the $k^{\text {th }}$ stress level. For any $t_{k}=t_{k, 1}<\cdots<t_{k, j}<\cdots<t_{k, M_{k}}=t_{k+1}, \quad j=1, \cdots, M_{k}-1$, we have independent but not identical random variables:

$$
\begin{aligned}
\Delta X_{i j k} & =X_{i(j+1) k}-X_{i j k} \\
& \sim N\left(\int_{t_{k, j}}^{t_{k, j+1}} \mu_{k}(t ; \theta) d t, \sigma_{k}^{2}\left(t_{k, j+1}-t_{k, j}\right)\right), \\
j & =1, \cdots, M_{k}-1 ; i=1, \cdots, N_{k} .
\end{aligned}
$$

According to Eq. (10), the likelihood function is given by Liao et al. [27]:

$$
\begin{aligned}
L(\theta) & =\prod_{1}^{k} \prod_{i=1}^{N_{k}} \prod_{j=1}^{M_{k}-1} \frac{1}{\sqrt{2 \pi \sigma_{k}^{2}\left(t_{k, j+1}-t_{k, j}\right)}} \\
& \cdot \exp \left(-\frac{\left(\left(x_{i(j+1) k}-x_{i j k}\right)-\int_{t}^{t_{k, j+1}} \mu_{k}(t ; \theta) d t\right)^{2}}{2 \sigma_{k}^{2}\left(t_{k, j+1}-t_{k, j}\right)}\right) .
\end{aligned}
$$

The likelihood function in Eq. (11) considers only the independent increment properties of the Brownian motion process. This method does not make a full use of the information, thus resulting in an inaccurate estimation. In our previous study, an estimation method was proposed which 
combines both the properties (P2) and (P3) into the likelihood function [48]. This method has been used for parameter estimation for mid-power white-light LED packages which were aged by means of CSADT. Similarly, the method can be also utilized for parameter estimation in step stress accelerated degradation test (SSADT) with a few modifications.

Firstly, for any $t_{k}=t_{k, 1}<t_{k, j}<\cdots<t_{k, q}<\cdots \leq t_{k+1}$, $j=2, \cdots, M_{k}-1, q=2, \cdots, M_{k} \quad$ and $j<q$, we have dependent random variables:

$$
\begin{aligned}
& X\left(t_{k, j}\right) \sim N\left(\int_{0}^{\tau_{k}+t_{k, j}} \mu_{k}(t ; \theta) d t, \sigma_{k}^{2}\left(\tau_{k}+t_{k, j}\right)\right) \\
& X\left(t_{k, q}\right) \sim N\left(\int_{0}^{\tau_{k}+t_{k, q}} \mu_{k}(t ; \theta) d t, \sigma_{k}^{2}\left(\tau_{k}+t_{k, q}\right)\right) .
\end{aligned}
$$

For simplicity, we assume the interdependency between $X\left(t_{j}\right)$ and $X\left(t_{q}\right)$ is negligible. With this assumption, the likelihood function is presented as:

$$
\begin{aligned}
& L(\theta)= \prod_{1}^{k} \prod_{i=1}^{N_{k}} \prod_{j=1}^{M_{k}-1} \frac{1}{2 \pi \sigma_{k}^{2} \sqrt{\left(\tau_{k}+t_{k, j+1}\right)\left(t_{k, j+1}-t_{k, j}\right)}} \\
& \cdot \exp \left(-\frac{\left(x_{i(j+1) k}-\int_{0}^{\tau_{k}+t_{k, j+1}} \mu_{k}(t ; \theta) d t\right)^{2}}{2 \sigma_{k}^{2}\left(\tau_{k}+t_{k, j+1}\right)}\right) \\
& \cdot \exp \left(-\frac{\left(\left(x_{i(j+1) k}-x_{i j k}\right)-\int_{t_{k, j}}^{t_{k, j+1}} \mu_{k}(t ; \theta) d t\right)^{2}}{2 \sigma_{k}^{2}\left(t_{k, j+1}-t_{k, j}\right)}\right) .
\end{aligned}
$$

The explicit form of $\mu_{k}(t ; \theta)$ is determined by the degradation models specified. For LED devices, it is well known that the lumen degradation can be described as $\Phi(t)=\Phi_{0} e^{-\beta t^{m}}$ [49]. Then, the lumen degradation rate, namely the drift coefficient $\mu_{k}(t ; \theta)$, can be obtained from the derivative of $\Phi(t)$. As a result, the time-dependent drift parameter of the Brownian motion is obtained as follows:

$$
\mu_{k}(t ; \theta)=-\beta_{k} m t^{m-1} \Phi_{0} e^{-\beta_{k} t^{m}}
$$

where $m$ is considered to be independent of stress levels, according to the theory of Tseng et al. [18]. This parameter is assumed to be 1 according to Ref. [4]. In this paper, the parameter $m$ will not be set as 1 , but will be fitted based on the degradation data. The parameter $\beta_{k}$ is dependent on the acceleration functions. The most widely used accelerated functions include Arrhenius model, Hallberg-Peck's model, and inverse power law model. These models can be employed for describing the effects of temperature, humidity-temperature, and electrical stress, respectively.

\section{EXPERIMENTS}

A 2-step SSADT experiment has been performed to illustrate the feasibility of the SSADT model. The LED packages used for experiments are shown in Fig. 2. The LED package was composed of five components: copper-based lead frames, molding compound package house, GaN-based LED chip, silicone/phosphor encapsulant, and gold wires which are used as electrical connections. These LED packages were first mounted onto metal-core printed circuit boards (MCPCBs). Each board contains 20 pieces of LED packages. After initial optical measurement, the samples were stored into a high temperature storage chamber for SSADT. At the first step, the ambient temperature was $130{ }^{\circ} \mathrm{C}$. After ageing for 500 hours, the ambient temperature was switched to $140^{\circ} \mathrm{C}$ and held until the end of the experiment. For comparison, 2 groups of LED samples were also separately placed into the high temperature storage chamber for CSADT. No electrical bias was applied during the ageing test. The detailed test conditions are shown in Table 1. During the ageing tests, the LED samples were taken out at every 100 hours interval for optical measurement.

It should be noticed that, in order to shorten the test duration, the ambient temperature is not selected according to maximum junction temperature that the LED manufacturer recommends (recommended maximum junction temperature is less than $125^{\circ} \mathrm{C}$ ). This implies that the data obtained in our experiments is not feasible for lifetime extrapolation for temperatures lower than $125{ }^{\circ} \mathrm{C}$, because the degradation mechanisms may be different from that obtained from LED packages aged at junction temperatures less than $125^{\circ} \mathrm{C}$. However, our analysis showed that the degradation mechanism is similar between $130^{\circ} \mathrm{C}$ and $140^{\circ} \mathrm{C}$, based on the theory proposed by Tseng et al. $[18,19]$. According to the theory, in the degradation model there exist some constants which indicate consistent degradation mechanisms for LED samples aged at different stress levels. In our model, the constant is parameter $m$, as mentioned in the previous paragraph. As a consequence, the data in our experiments can be used for SSADT modeling, as will be discussed in the following paragraph. Furthermore, the data can be also used for lifetime extrapolation if the operating temperature is between $130{ }^{\circ} \mathrm{C}$ and $140{ }^{\circ} \mathrm{C}$. 


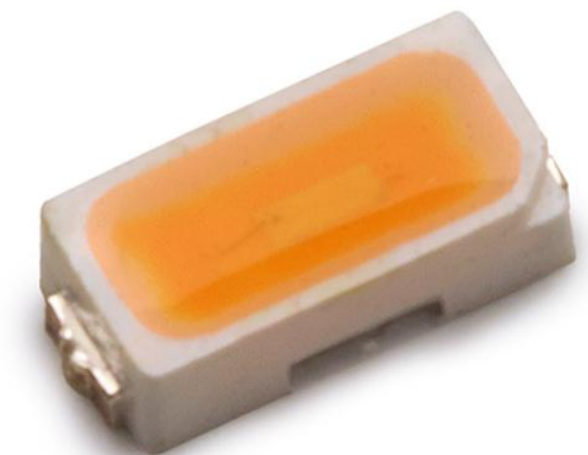

(a) LED package

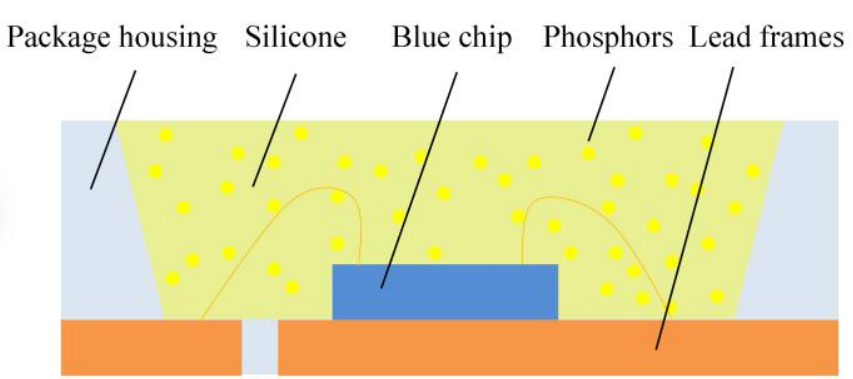

(b) LED structure

Fig. 2 A type of white-light LED package and its structure

Table 1 Stress conditions for SSADT and CSADT

\begin{tabular}{cccccc}
\hline Test type & Sample & Sample size & Ambient temperature & Bias & Ageing duration \\
SSADT & Group 1 & 20 units & $130{ }^{\circ} \mathrm{C}$ & No bias & 500 hours \\
SSADT & Group 1 & 20 units & $140{ }^{\circ} \mathrm{C}$ & No bias & 500 hours \\
CSADT & Group 2 & 20 units & $130{ }^{\circ} \mathrm{C}$ & No bias & 500 hours \\
CSADT & Group 3 & 20 units & $140^{\circ} \mathrm{C}$ & No bias & 500 hours \\
\hline
\end{tabular}

\section{RESULTS AND DISCUSSION}

After finishing all the tests, the lumen outputs were normalized at 0 hour for each individual sample, as shown in Fig. 3 (a) and (b). It has been found that the lumen degradation could be approximated by the exponential model. For the data obtained from SSADT, according to the analysis method provided in the IES standard [4], the activation energy $E_{a}$ is calculated as $0.59 \mathrm{eV}$, which is higher than that reported in [46], where the maximum junctions temperature is lower than $130{ }^{\circ} \mathrm{C}$.

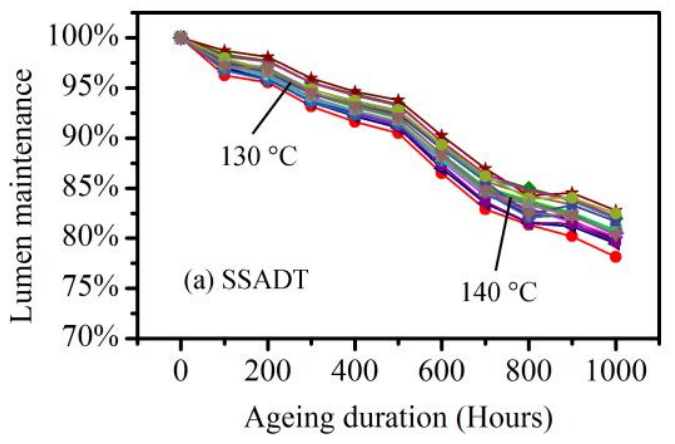

However, when we calculate the independent parameter $m$, according to model presented in $[18,19]$ :

$\Phi(t)=\Phi_{0} e^{-\beta t^{m}}$

it has been found that the values of the independent parameter $m$ is calculated as 0.808 and 0.619 for both the SSADT data set collected from 0 hour to 500 hours, and data set collected from 500 hours and 1000 hours. The difference between the two independent parameters is $\Delta m=0.189<0.2$. According to the theory in Ref. [19], the degradation mechanisms can be regarded as consistent during the SSADT test.

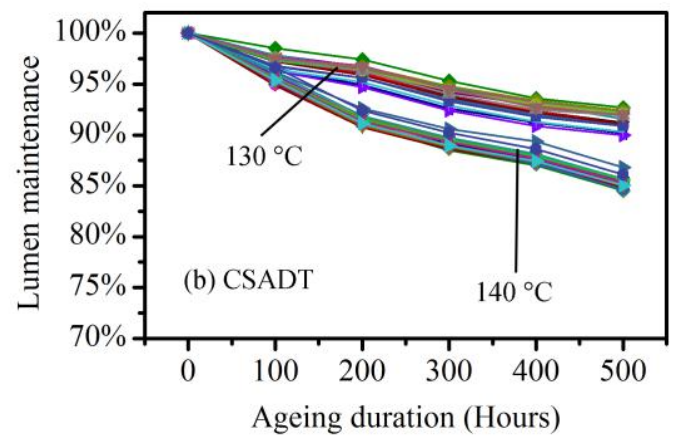

Ageing duration (Hours)
The degradation mechanisms were further confirmed by using failure analysis. As can be seen in Fig. 4, I-V characteristics show no obvious difference between the fresh samples and aged samples, indicating that the blue dies did not deteriorate during the high temperature storage tests. However, yellowing of the package housing was found on the aged samples, which are stressed under either CSADT or SSADT. Due to longer ageing duration, the samples aged under SSADT show more significant yellowing than those aged under CSADT, as presented in Fig. 5. Due to lack of equipments, we could not analyze the degradation of the silicone encapsulant lead frames, and phosphors. However, our previous research already showed very good performance for these two materials
[50]. As a result, it was concluded that the major degradation was the yellowing of the package housing.

Normality test was also performed for the degradation data obtained from SSADT test. The analysis was completed by using the commercial statistical analysis code Minitab. A p-value larger than 0.1 will indicate that a data set passes the normality test. As shown in Tables 2 and 3, most of the data sets follow normal distributions, indicating that Brownian motion process can be used for the degradation modeling.

As a consequence, the lumen degradation model for the SSADT data can be written as in Eq. (16): 


$$
X(t)=\left\{\begin{array}{l}
\Phi_{10} \exp \left(-\alpha_{1} \mathrm{t}^{m}\right)+\sigma_{1} B(t), \quad T_{a}=130{ }^{\circ} \mathrm{C} \\
\Phi_{20} \exp \left[-500^{m} \alpha_{1}-\alpha_{2}(\mathrm{t}-500)^{m}\right] \\
+\sigma_{2} B\left(\frac{\alpha_{1}^{1 / m}}{\alpha_{2}^{1 / m}} \cdot 500+t-500\right),
\end{array} .\right.
$$

On the other hand, the lumen degradation model for the CSADT data is written as in Eq. (17):

$$
X(t)=\left\{\begin{array}{ll}
\Phi_{10} \exp \left(-\alpha_{1} \mathrm{t}^{m}\right)+\sigma_{1} B(t) & T_{a}=130{ }^{\circ} \mathrm{C} \\
\Phi_{20} \exp \left(-\alpha_{2} \mathrm{t}^{m}\right)+\sigma_{2} B(t) & T_{a}=140{ }^{\circ} \mathrm{C}
\end{array} .\right.
$$

The unknown parameters in Eq. (16) and (17) include $\Phi_{10}, \Phi_{20}, \alpha_{1}, \alpha_{2}, \sigma_{1}, \sigma_{2}, m$. Based on Eq. (16) and (17), the unknown parameters can be estimated according to the likelihood function in Eq. (13). It is found that the likelihood function in Eq. (13) is very complicated and analytically intractable. Therefore, Bayesian Markov chain Monte Carlo simulation (MCMC) was performed. More details about the algorithm are referred to Ref. [51]. After 200,000 iterations, all parameters became convergent. The results are shown in Table 4 and 5.

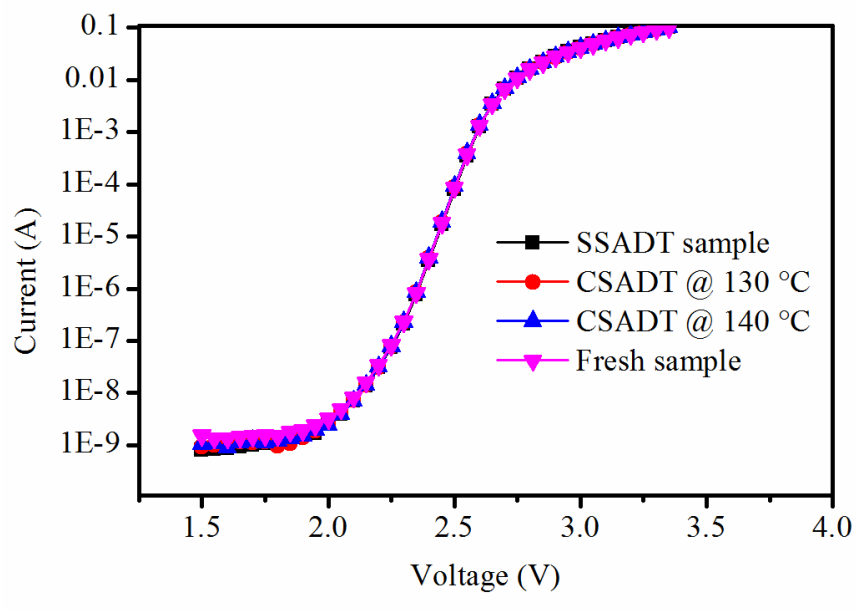

Fig. 4 I-V characteristics of fresh and aged samples

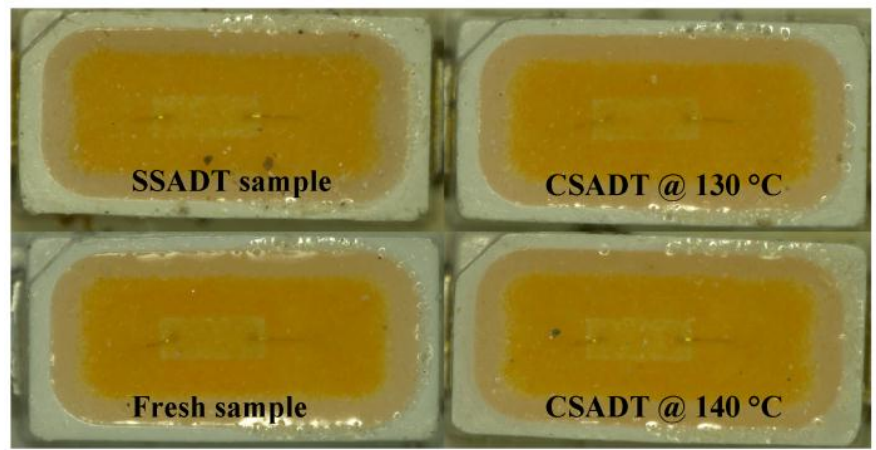

Fig. 5 Yellowing of the package housing

\begin{tabular}{|c|c|c|c|c|c|c|}
\hline $\begin{array}{l}\text { Readouts } \\
\text { (Hours) }\end{array}$ & 0 & 100 & 200 & 300 & 400 & 500 \\
\hline P-value & 1 & 0.523 & 0.381 & 0.296 & 0.402 & 0.551 \\
\hline $\begin{array}{l}\text { Readouts } \\
\text { (Hours) }\end{array}$ & 500 & 600 & 700 & 800 & 900 & 1000 \\
\hline P-value & 0.551 & 0.678 & 0.496 & 0.783 & 0.231 & 0.38 \\
\hline \multicolumn{7}{|c|}{ Table 3 Normality test for normalized lumen degradation between two adjacent readouts } \\
\hline $\begin{array}{l}\text { Readouts } \\
\text { (Hours) }\end{array}$ & $\mathbf{0}$ & 0 - 100 & $100-200$ & $200-300$ & $300-400$ & $400-500$ \\
\hline P-value & 1 & 0.523 & 0.402 & 0.888 & 0.461 & 0.532 \\
\hline $\begin{array}{l}\text { Readouts } \\
\text { (Hours) }\end{array}$ & $400-500$ & $500-600$ & $600-700$ & $700-800$ & $800-900$ & $900-1000$ \\
\hline P-value & 0.532 & 0.38 & 0.113 & 0.01 & 0.005 & 0.839 \\
\hline \multicolumn{7}{|c|}{ Table 4 Parameter estimation results of SSADT } \\
\hline Parameter & mean & $\begin{array}{l}\text { standard } \\
\text { deviation }\end{array}$ & MC error & $2.50 \%$ & median & $\mathbf{9 7 . 5 0 \%}$ \\
\hline$\alpha_{1}$ & $9.81 \mathrm{E}-04$ & $1.01 \mathrm{E}-04$ & $3.84 \mathrm{E}-06$ & 8.01E-04 & $9.77 \mathrm{E}-04$ & $1.20 \mathrm{E}-03$ \\
\hline$\alpha_{2}$ & $1.60 \mathrm{E}-03$ & $1.60 \mathrm{E}-04$ & $6.12 \mathrm{E}-06$ & $1.31 \mathrm{E}-03$ & $1.59 \mathrm{E}-03$ & 0.001937 \\
\hline $\mathrm{m}$ & 0.7128 & $1.62 \mathrm{E}-02$ & $6.35 \mathrm{E}-04$ & 0.6804 & 0.7126 & 0.744 \\
\hline$\Phi_{10}$ & 1.001 & 0.001469 & $2.01 \mathrm{E}-05$ & 0.9983 & 1.001 & 1.004 \\
\hline$\Phi_{20}$ & 0.9967 & 0.003351 & $2.33 \mathrm{E}-05$ & 0.9901 & 0.9967 & 1.003 \\
\hline$\sigma_{1}$ & $5.45 \mathrm{E}-04$ & $2.78 \mathrm{E}-05$ & $1.36 \mathrm{E}-07$ & 4.94E-04 & $5.44 \mathrm{E}-04$ & $6.03 \mathrm{E}-04$ \\
\hline$\sigma_{2}$ & $6.73 \mathrm{E}-04$ & $3.43 \mathrm{E}-05$ & $1.35 \mathrm{E}-07$ & $6.09 \mathrm{E}-04$ & $6.71 \mathrm{E}-04$ & 7.44E-04 \\
\hline \multicolumn{7}{|c|}{ Table 5 Parameter estimation results of CSADT } \\
\hline parameter & mean & $\begin{array}{l}\text { standard } \\
\text { deviation }\end{array}$ & MC error & $2.50 \%$ & median & $\mathbf{9 7 . 5 0 \%}$ \\
\hline$\alpha_{1}$ & 8.75E-04 & $6.21 \mathrm{E}-05$ & $2.37 \mathrm{E}-06$ & 7.59E-04 & $8.73 \mathrm{E}-04$ & $1.00 \mathrm{E}-03$ \\
\hline
\end{tabular}

Table 2 Normality test for normalized lumen output at each readout 


\begin{tabular}{lllllll}
\hline$\alpha_{2}$ & $1.59 \mathrm{E}-03$ & $1.09 \mathrm{E}-04$ & $4.25 \mathrm{E}-06$ & $1.39 \mathrm{E}-03$ & $1.59 \mathrm{E}-03$ & $1.81 \mathrm{E}-03$ \\
$\mathrm{~m}$ & $7.43 \mathrm{E}-01$ & $1.09 \mathrm{E}-02$ & $4.28 \mathrm{E}-04$ & $7.23 \mathrm{E}-01$ & $7.43 \mathrm{E}-01$ & $7.65 \mathrm{E}-01$ \\
$\Phi_{10}$ & $1.00 \mathrm{E}+00$ & $1.29 \mathrm{E}-03$ & $1.88 \mathrm{E}-05$ & $9.98 \mathrm{E}-01$ & $1.00 \mathrm{E}+00$ & $1.00 \mathrm{E}+00$ \\
$\Phi_{20}$ & $9.99 \mathrm{E}-01$ & $1.60 \mathrm{E}-03$ & $2.88 \mathrm{E}-05$ & $9.96 \mathrm{E}-01$ & $9.99 \mathrm{E}-01$ & $1.00 \mathrm{E}+00$ \\
$\sigma_{1}$ & $4.89 \mathrm{E}-04$ & $2.49 \mathrm{E}-05$ & $6.26 \mathrm{E}-08$ & $4.44 \mathrm{E}-04$ & $4.88 \mathrm{E}-04$ & $5.41 \mathrm{E}-04$ \\
$\sigma_{2}$ & $5.77 \mathrm{E}-04$ & $2.93 \mathrm{E}-05$ & $8.73 \mathrm{E}-08$ & $5.23 \mathrm{E}-04$ & $5.76 \mathrm{E}-04$ & $6.38 \mathrm{E}-04$ \\
\hline
\end{tabular}

Table 6 Parameter comparison between SSADT and CSADT

\begin{tabular}{llllllll}
\hline Temperature & \multicolumn{3}{c}{$\mathbf{1 3 0}{ }^{\circ} \mathbf{C}$} & \multicolumn{3}{c}{$\mathbf{1 4 0}^{\circ} \mathbf{C}$} \\
\hline Parameter & $\alpha_{1}$ & $\Phi_{10}$ & $\sigma_{1}$ & $\mathrm{~m}$ & $\alpha_{2}$ & $\Phi_{20}$ & $\sigma_{2}$ \\
SSADT & $9.81 \mathrm{E}-04$ & $1.00 \mathrm{E}+00$ & $5.45 \mathrm{E}-04$ & $7.13 \mathrm{E}-01$ & $1.60 \mathrm{E}-03$ & $9.97 \mathrm{E}-01$ & $6.73 \mathrm{E}-04$ \\
CSADT & $8.75 \mathrm{E}-04$ & $1.00 \mathrm{E}+00$ & $4.89 \mathrm{E}-04$ & $7.43 \mathrm{E}-01$ & $1.59 \mathrm{E}-03$ & $9.99 \mathrm{E}-01$ & $5.77 \mathrm{E}-04$ \\
Error & $12.11 \%$ & $0.00 \%$ & $11.40 \%$ & $-4.12 \%$ & $0.38 \%$ & $-0.25 \%$ & $16.49 \%$ \\
\hline
\end{tabular}

For comparison, the mean of parameters in Table 4 and 5 were re-arranged as shown in Table 6. The error between SSADT and CSADT has been calculated by dividing the parameter difference for each parameter obtained by CSADT. For instance, in Table 6, the error of the parameter $\sigma_{1}$ is calculated as $(0.000981-0.000875) \div 0.000875=12.11 \%$. As shown in Table 6, except for parameter $\sigma_{2}$, the estimated parameters obtained from SSADT data are very close to that obtained from CSADT data, demonstrating the feasibility of our SSADT model. Fig. 6 shows the lumen degradation fitted by our SSADT model. The variations given by the SSADT model match the variations of the experiment data very well. According to Eq. (4), the cumulative distribution functions (CDF) were plotted based on the parameters in Table 6, which is shown in Fig. 7. The mean time to failure (MTTF) calculated by using the data obtained from SSADT and CSADT was also given, as presented in Table 7. Both testing methods show similar MTTF at the same storage temperature. The slight difference of the MTTF between both testing methods may be due to the unbalance test duration between CSADT and SSADT. Prolonging the CSADT ageing time to 1000 hours (testing duration is 1000 hours in SSADT) should be performed to minimize the difference.

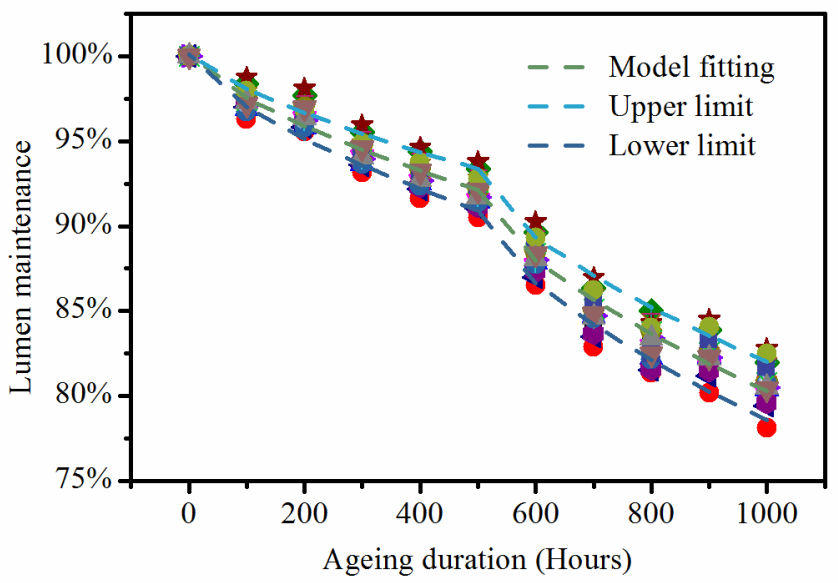

Fig. 6 Lumen degradation fitted by SSADT model

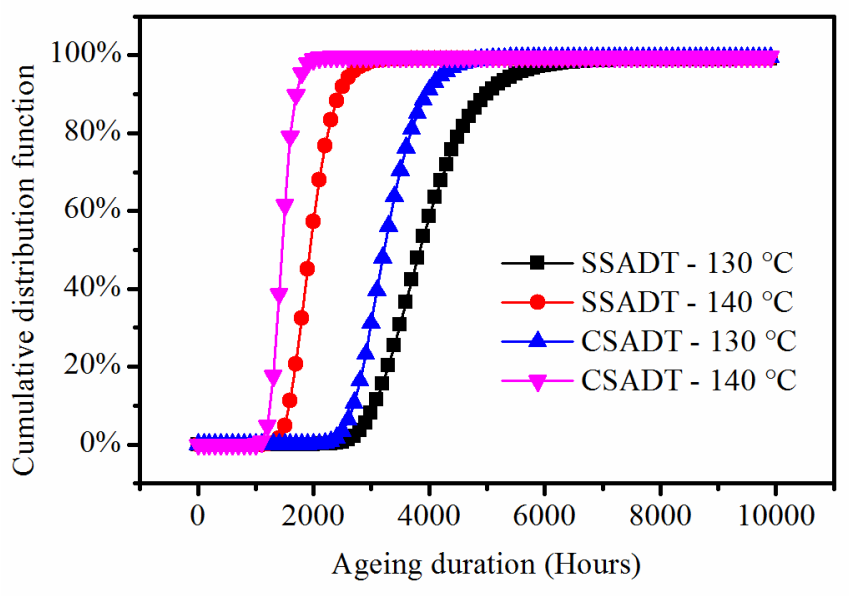

Fig. 7 The cumulative failure function of LED devices at different stress conditions

Table 7 Mean time to failure of LEDs at different conditions

\begin{tabular}{ccccc}
$\begin{array}{c}\text { Stress } \\
\text { condition }\end{array}$ & $\begin{array}{c}\text { SSADT }- \\
\mathbf{1 3 0}^{\circ} \mathbf{C}\end{array}$ & $\begin{array}{c}\text { SSADT - } \\
\mathbf{1 4 0}^{\circ} \mathbf{C}\end{array}$ & $\begin{array}{c}\text { CSADT } ~ \\
\mathbf{1 3 0}^{\circ} \mathbf{C}\end{array}$ & $\begin{array}{c}\text { CSADT - } \\
\mathbf{1 4 0}^{\circ} \mathbf{C}\end{array}$ \\
\hline $\begin{array}{c}\text { MTTF } \\
\text { (Hours) }\end{array}$ & 3893 & 1962 & 3257 & 1458.5 \\
\hline
\end{tabular}

It is worth noticing that, in this section, we utilized LED packages as an example to verify the feasibility of the model. However, this model is not limited to the application of LEDs, it can be also applied to more broad applications, including electronic products, automobiles, aerospace prototypes, etc, as long as the explicit form of $\mu_{k}(t ; \theta)$ is given. The SSADT is especially suitable for reliability tests in which samples are very expensive and rare, or resources are limited.

\section{CONCLUSION}

In this paper, an SSADT model was proposed by using a modified Brownian motion process. The theory of Brownian motion process, the modeling of the SSADT, as well as the parameter estimation has been presented. In order to indicate the feasibility of the SSADT model, a 2-step SSADT has been performed on one group of mid-power LED packages. At the same time, two groups of LED packages were also respectively aged by using the traditional CSADT. Parameters obtained from both SSADT and CSADT data show comparable results, 
indicating the feasibility of the proposed SSADT model. The experiment also indicates that SSADT can be used as an alternative instead of CSADT, because it enables comparable estimation accuracy while using smaller sample size, and less test resources.

\section{ACKNOWLEDGMENT}

The author Jianlin Huang thank for the supports of Bobby and Candy from Integrated service technology.

\section{REFERENCES}

[1] J. Huang, et al., "Degradation Mechanisms of Mid-power White-light LEDs under High Temperature-Humidity Conditions," IEEE Trans. Device Mater. Reliab., vol. 15, no. 2, pp. 220-228, Jun. 2015.

[2] M. Buffolo, et al., "Long-term degradation mechanisms of mid-power LEDs for lighting applications," Microelectron. Reliab., vol. 55, no. 9, pp. 1754-1758, 2015.

[3] I. T. P. Committee, "Approved Method: Measuring Lumen Maintenance of LED Light Sources," IESNA LM-80-08. Illuminating Engineering Society of North America. New York2008.

[4] I. E. S. o. N. America, "Projecting long term lumen maintenance of LED light sources," ed: Illuminating Engineering Society New York (NY), 2011.

[5] W. Nelson, "Analysis of performance-degradation data from accelerated tests," IEEE Trans. Reliab., vol. 30, no. 2, pp. 149-155, Jun. 1981.

[6] C.-C. Tsai and C.-T. Lin, "Lifetime Inference for Highly Reliable Products Based on Skew-Normal Accelerated Destructive Degradation Test Model," Reliability, IEEE Transactions on, vol. 64, no. 4, pp. 1340-1355, 2015

[7] Y. Wang, et al., "Optimal design of constant stress accelerated degradation test plan with multiple stresses and multiple degradation measures," Proceedings of the Institution of Mechanical Engineers, Part O: Journal of Risk and Reliability, vol. 229, no. 1, pp. 83-93, 2015.

[8] C. J. Lu and W. Q. Meeker, "Using degradation measures to estimate a time-to-failure distribution," Technometrics, vol. 35, no. 2, pp. 161-174, May 1993.

[9] C. J. Lu, W. Q. Meeker and L. A. Escobar, "A comparison of degradation and failure-time analysis methods for estimating a time-to-failure distribution," Stat. Sin., vol. 6, no. 3, pp. 531-546, Jul. 1996.

[10] M. A. Freitas, et al., "Reliability assessment using degradation models: Bayesian and classical approaches," Pesqui. Oper., vol. 30, no. 1, pp. 194-219, 2010

[11] J. Fan, K.-C. Yung and M. Pecht, "Lifetime estimation of high-power white LED using degradation-data-driven method," IEEE Trans. Device Mater. Reliab., vol. 12, no. 2, pp. 470-477, Jun. 2012.

[12] G. Whitmore, "Estimating degradation by a Wiener diffusion process subject to measurement error," Lifetime Data Anal., vol. 1, no. 3, pp. 307-319, 1995.

[13] C. Park and W. Padgett, "Accelerated degradation models for failure based on geometric Brownian motion and gamma processes," Lifetime Data Anal., vol. 11, no. 4, pp. 511-527, Dec. 2005

[14] T.-R. Tsai, et al., "Inference from lumen degradation data under Wiener diffusion process," IEEE Trans. Reliab., vol. 61, no. 3, pp. 710-718, Jul. 2012

[15] Z.-S. Ye, N. Chen and Y. Shen, "A new class of Wiener process models for degradation analysis," Reliab. Eng. Syst. Saf., vol. 139, pp. 58-67, Jul. 2015.

[16] C. Zhang, et al., "Reliability demonstration methodology for products with Gamma Process by optimal accelerated degradation testing," Reliab. Eng. Syst. Saf., vol. 142, pp. 369-377, 2015.

[17] Z. S. Ye and M. Xie, "Stochastic modelling and analysis of degradation for highly reliable products," Applied Stochastic Models in Business and Industry, vol. 31, no. 1, pp. 16-32, 2015.
The author Daoguo Yang would like to thank the National Science Foundation of China for the support (Grant No. 51366003).

This work has been accomplished within EMRP JRP ENG62 MESaIL which was carried out with funding by the European Union. The EMRP is jointly funded by the EMRP participating countries within EURAMET and the European Union.

S.-T. Tseng and Z.-C. Wen, "Step-stress accelerated degradation analysis for highly reliable products," J. Qual. Technol., vol. 32, no. 3, pp. 209-216, Jul. 2000

M. Cai, et al., "Step-stress accelerated testing of high-power LED lamps based on subsystem isolation method," Microelectron. Reliab., vol. 55, no. 9, pp. 1784-1789, Aug. 2015.

[20] C.-H. Hu, M.-Y. Lee and J. Tang, "Optimum step-stress accelerated degradation test for Wiener degradation process under constraints," European Journal of Operational Research, vol. 241, no. 2, pp. 412-421, 2015.

[21] M. Cai, et al., "Accelerated testing method of LED luminaries," in Proc. EuroSimE, Cascais, 2012, pp. 1/6-6/6.

[22] F. Haghighi and S. J. Bae, "Reliability Estimation from Linear Degradation and Failure Time Data With Competing Risks Under a Step-Stress Accelerated Degradation Test," IEEE Trans. Reliab., vol. 64, no. 3, pp. 960-971 Jul. 2015.

[23] C. Wei-Wei, et al., "The degradation mechanism of an AlGaN/GaN high electron mobility transistor under step-stress," Chin. Phys. B, vol. 22 , no. 10, p. 107303, 2013.

[24] Y. Jun, X. Mingge and W. ZHONG, "Research of Step-down Stress Accelerated Degradation Data Assessment Method of a Certain Type of Missile Tank," Chin. J. Aeronaut., vol. 25, no. 6, pp. 917-924, Dec. 2012

[25] Y. J. Z. Weiqiang, "Assessing step-down stress accelerated degradation data via nonlinear accelerated model," J. B. Univ. Aeronaut. Astronaut., vol. 12, no. 12, pp. 1475-1478, Dec. 2011.

[26] K. A. Doksum and A. Hbyland, "Models for variable-stress accelerated life testing experiments based on Wiener processes and the inverse gaussian distribution," Technometrics, vol. 34, no. 1, pp. 74-82, Mar. 1992.

[27] C.-M. Liao and S.-T. Tseng, "Optimal design for step-stress accelerated degradation tests," IEEE Trans. Reliab., vol. 55, no. 1, pp. 59-66, Mar. 2006.

[28] C.-C. Tsai, S.-T. Tseng and N. Balakrishnan, "Optimal Design for Degradation Tests Based on Gamma Processes With Random Effects," IEEE Trans. Reliab., vol. 61, no. 2, pp. 604-613, May 2012.

[29] A. Molini, et al., "First passage time statistics of Brownian motion with purely time dependent drift and diffusion," Physica A, vol. 390, no. 11, pp. 1841-1852, Jun. 2011.

[30] R. Harman and F. Stulajter, "Optimality of equidistant sampling designs for the Brownian motion with a quadratic drift," J. Stat. Plan. Infer., vol. 141, no. 8, pp. 2750-2758, Aug. 2011.

[31] E. Urdapilleta, "Series solution to the first-passage-time problem of a Brownian motion with an exponential time-dependent drift," $J$. Phys. A-Math. Theor., vol. 45, no. 18, May 2012.

[32] J. Li, "Some limit results for probabilities estimates of Brownian motion with polynomial drift," Indian J. Pure Appl. Math., vol. 41, no. 3 , pp. 425-442, Jun. 2010

[33] E. Urdapilleta, "Survival probability and first-passage-time statistics of a Wiener process driven by an exponential time-dependent drift," Phys. Rev. E, vol. 83, no. 2, Feb. 2011.

[34] X.-S. Si, et al., "Remaining useful life estimation based on a nonlinear diffusion degradation process," IEEE Trans. Reliab., vol. 61, no. 1, pp. 50-67, Jan. 2012.

[35] X.-S. Si, et al., "A Wiener-process-based degradation model with a recursive filter algorithm for remaining useful life estimation," Mech. Syst. Signal Proc., vol. 35, no. 1, pp. 219-237, Feb. 2013.

[36] W. Zhao-Qiang, et al., "An Additive Wiener Process-Based Prognostic Model for Hybrid Deteriorating Systems," IEEE Trans. Reliab., vol. 63, no. 1, pp. 208-222, Mar. 2014. 
[37] X. Wang, N. Balakrishnan and B. Guo, "Residual life estimation based on a generalized Wiener degradation process," Reliab. Eng. Syst. Saf., vol. 124, pp. 13-23, Apr. 2014.

[38] X. Wang, B. Guo and Z. Cheng, "Residual life estimation based on bivariate Wiener degradation process with time-scale transformations," J. Stat. Comput. Simul., vol. 84, no. 3, pp. 545-563, Oct. 2014.

[39] Y. Wang, Z.-S. Ye and K.-L. Tsui, "Stochastic evaluation of magnetic head wears in hard disk drives," IEEE Trans. Magn., vol. 50, no. 5, pp. 1-7, 2014.

[40] H. Zeyi, et al., "Remaining Useful Life Prediction for a Nonlinear Heterogeneous Wiener Process Model With an Adaptive Drift," IEEE Trans. Reliab., vol. 64, no. 2, pp. 687-700, Jun. 2015.

[41] J. Y. Chiang, Y. Lio and T. R. Tsai, "Degradation Tests Using Geometric Brownian Motion Process for Lumen Degradation Data," Qual. Reliab. Eng. Int., vol. 31, no. 8, pp. 1797-1806, 2015.

[42] C.-Y. Peng and S.-T. Tseng, "Progressive-stress accelerated degradation test for highly-reliable products," IEEE Trans. Reliab., vol. 59, no. 1, pp. 30-37, Mar. 2010.

[43] X. Wang, et al., "Real - time Reliability Evaluation with a General Wiener Process - based Degradation Model," Qual. Reliab. Eng. Int., vol. 30, no. 2, pp. 205-220, Mar. 2014.

[44] C. M. Tan and P. Singh, "Time Evolution Degradation Physics in High Power White LEDs Under High Temperature-Humidity Conditions," IEEE Trans. Device Mater. Reliab., vol. 14, no. 2, pp. 742-750, Jun. 2014.

[45] C. Guo, et al., "Failure-mechanism identification method in acceleratedtesting," Chin. J. Semicond., vol. 27, no. 3, pp. 560-563, Mar. 2006.

[46] S. Koh, et al., "Product Level Accelerated Lifetime Test for Indoor LED Luminaires," in Proc. EuroSimE, Wroclaw, 2013, pp. 1-6.

[47] W. Nelson, "Accelerated life testing-step-stress models and data analyses," IEEE Trans. Reliab., vol. 29, no. 2, pp. 103-108, Jun. 1980.

[48] J. Huang, et al., "Degradation modeling of mid-power white-light LEDs by using Wiener process," Opt. Express, vol. 23, no. 15, pp. A966-A978, 2015.

[49] S. Yamakoshi, et al., "Degradation of high - radiance Ga 1-x Al x As LED' s," Appl. Phys. Lett., vol. 31, no. 9, pp. 627-629, Nov. 1977.

[50] J. Huang, et al., "Rapid degradation of mid-power white-light LEDs in saturated moisture conditions," IEEE Trans. Device Mater. Reliab., vol. 15, no. 4, pp. 478-485, 2015.

[51] D. J. Lunn, et al., "WinBUGS-a Bayesian modelling framework: concepts, structure, and extensibility," Stat. Comput., vol. 10, no. 4, pp. 325-337, Oct. 2000. 